\title{
The Intermediate Scale Branch of the Landscape
}

\author{
M. Dine \\ Santa Cruz Institute for Particle Physics, Santa Cruz CA 95064 \\ Email: dine@scipp.ucsc.edu
}

\begin{abstract}
Three branches of the string theory landscape have plausibly been identified. One of these branches is expected to exhibit a roughly logarithmic distribution of supersymmetry breaking scales. The original KKLT models are in this class. We argue that certain features of the KKLT model are generic to this branch, and that the resulting phenomenology depends on a small set of discrete choices. As in the MSSM, the weak scale in these theories is tuned; a possible explanation is selection for the dark matter density.
\end{abstract}




\section{Contents}

1. Introduction: Supersymmetry Breaking in the Landscape 1

2. The KKLT Picture 5

3. KKLT At Low Energies 7

3.1 Structure of Soft Breakings

3.2 Light Kahler Moduli; Hidden Sector on D3 Branes 8

3.3 Hidden Sector on $D 7$ Branes 10

4. Phenomenology and Cosmology 10

4.1 Cosmology 11

4.2 Phenomenology

5. Conclusions: Selection effects, the little hierarchy, and other issues 12

\section{Introduction: Supersymmetry Breaking in the Landscape}

Implicit in traditional questions of naturalness is the notion that there is a distribution of possible theories of elementary particles. The possible existence, in string theory, of an exponentially large number of stable and metastable vacua without supersymmetry or with $N=1$ supersymmetry in four dimensions, the "landscape" [1, 2, 3, 4], provides a realization of this idea. Much is already known about the statistics of these states [5, 6, 7, 8, 9], and it is possible to assess the tunings required to understand the values of parameters such as the cosmological constant and the weak scale. It is even possible to make some cautious statements about correlations which might lead to experimental predictions. There are many uncertainties, and any discussion of the landscape must be viewed as tentative. For example, recently, an analysis has appeared that suggests there might be infinite numbers of four dimensional stable or metastable states in string theory, with all moduli fixed and with no more than four supercharges. These are even claimed to be accessible to weak coupling methods 10]. If correct, and if these states are physically relevant, many of our notions of vacuum statistics are, at best, naive. We will comment on these issues briefly in our conclusions, but the discussion of this paper will be predicated on the assumption that the number of relevant states in the landscape is finite and conventional statistical ideas can be applied.

Three branches of the landscape have been clearly identified $[8]$. They are distinguished by their distributions of supersymmetry breaking scales. On one branch, which we will refer to as the "broken supersymmetric branch", the bulk of the states have supersymmetry 
broken at very high energies. One can make reliable statements on this branch only for small supersymmetry breaking scale, $m_{3 / 2} \ll M_{p}$. In this regime the distribution behaves, for small cosmological constant, $\Lambda<\Lambda_{o}$, as [7, 9

$$
\int d m_{3 / 2} P\left(m_{3 / 2}\right)=\Lambda_{o} \int d m_{3 / 2}^{2} m_{3 / 2}^{10}
$$

where we use units where the Planck scale is set equal to one. On the second branch, the "intermediate scale branch", the distribution of supersymmetry-breaking scales is expected to be roughly logarithmic [8]:

$$
\int P\left(m_{3 / 2}\right) d m_{3 / 2}=\int \frac{d m_{3 / 2}^{2}}{m_{3 / 2}^{2}} \ln \left(m_{3 / 2}\right)
$$

On the third branch, the "low scale branch," the scale of supersymmetry breaking tends to be very small[ [8],

$$
\int P\left(m_{3 / 2}\right) d m_{3 / 2}=\int \frac{d m_{3 / 2}^{2}}{m_{3 / 2}^{4}}
$$

In tree level analyses, e.g. of IIB theories on orientifolds of Calabi-Yau spaces, the first branch corresponds to stationary points of the action with broken supersymmetry; the second branch to points with unbroken supersymmetry and negative cosmological constant (non-vanishing superpotential, $W$ ); the third branch to states with unbroken supersymmetry and vanishing cosmological constant at tree level. Since one expects that $N=1$ supersymmetry is often dynamically broken, these classical distinctions are not sharp. However, the statistics we have described are likely to be features of any final formulation of the landscape.

Indeed, these statistics appear to be robust (at least if the number of states is finite). They were first uncovered in studies of particular classes of string models, but they follow from very modest assumptions: the existence of a dense set of states in a particular range of parameters and the absence of singularities in the distribution of parameters, apart from those which can be understood on symmetry grounds.

Without a detailed understanding of microphysics, however, there are questions which one cannot address. For the landscape, the most important of these are the relative populations of these branches, and cosmological or other effects which might select one branch over another. At present, one can at best offer only speculative arguments why nature might find itself on one or another of these branches:

1. The non-supersymmetric branch might be favored simply because there might be vastly more non-supersymmetric than supersymmetric states. A number of constructions of such states have been exhibited[12, 13, 14]. Because of the need to impose a cutoff on the supersymmetry breaking scale to control the calculation, it is not presently possible to reliably count the states or ascertain their statistics. For cutoff slightly below the fundamental scale, it seems that the numbers of supersymmetric and non-supersymmetric states are comparable. If there are vastly more supersymmetric than non-supersymmetric states, than in the bulk of these states, it is unlikely 
that there is any fundamental small parameter. The supersymmetry breaking scale, internal radii, and so on are all likely to be of order one. In states with small cosmological constant (in which it makes sense to speak of a low energy theory), there will still be a distribution of low energy parameters, most of which, presumably, must be determined anthropically. It is hard to understand how such a picture can be consistent with the facts of the Standard Model 15.

2. The number of non-supersymmetric states might be highly suppressed, as suggested by the following simple-minded stability argument. Supersymmetric states are stable, so states with very small SUSY breaking are likely to be highly metastable. For nonsupersymmetric states, the situation is potentially quite different. The arguments of [7, 9] establish criteria for the counting of local minima, but global questions are more challenging. The typical non-supersymmetric state with small, positive cosmological constant is surrounded (in the lattice of fluxes) by other states, presumably roughly half of which typically have negative cosmological constant. The standard Coleman-DeLuccia analysis 16] would lead us to expect that to some of these states the decay amplitude vanishes, while to others there is a decay to an open universe which experiences a big crunch. In the absence of small parameters, one might guess that a typical state with small cosmological constant and badly broken symmetry could decay to $50 \%$ of its neighbors. So if our would-be De Sitter vacuum has, say, 300 neighbors, then the probability that it does not undergo rapid decay - that it can even be thought of as a state - is of order $(1 / 2)^{300}$. It is, of course, possible that there are, in the landscape, so many more non-supersymmetric stationary points than supersymmetric ones that even this suppression is not important. It is also possible that these sorts of naive field theory ideas are not relevant[11].

3. The discussion above raises the possibility that the number of supersymmetric states might not be so much smaller than the number of non-supersymmetric states. In this case, due to the logarithmic distribution of scales, a low scale of supersymmetry breaking is reasonably probable. The value of the weak scale might then be a plausible accident. This is just the usual argument for naturalness of low energy supersymmetry breaking. There could well also be selection effects which prefer a small weak (and therefore susy) breaking scale. If there are comparable numbers of states on the supersymmetric and non-supersymmetric branches, then there are vastly more states on the former with a small value of the weak scale - again, this is a version of the usual naturalness problem. Even if there are far more non-supersymmetric than supersymmetric states, it is conceivable that inflation and dark matter might might favor the supersymmetric branch (we will discuss these possibilities at greater length below). A few phenomenological facts (coupling unification, for example) provide at least a hint that this branch may be favored.

4. States on the low energy branch arise when there is a (dynamically broken) Rsymmetry and supersymmetry. These states are inevitably less numerous than states without such symmetries [8, 17, 9], and recent studies have shown that this suppres- 
sion can be quite substantial[18, 19]. One other troubling feature of this branch is that the vast majority of states have moduli, which are only fixed as a result of supersymmetry breaking. If the scale of supersymmetry breaking is low, as suggested by the distribution above, then these moduli lead to significant cosmological problems with no obvious resolution. Note the $R$ symmetries under consideration here are symmetries under which the superpotential transforms non-trivially; conventional $R$-parity is not in this category. The analysis of [19] indicates that states with $Z_{2}$ $R$-parity like symmetries are not highly suppressed. These would be compatible with understanding the absence of proton decay in the intermediate branch.

It appears difficult to fully resolve these questions in the near future. The nonsupersymmetric branch is particularly problematic, since the supersymmetry breaking being large in virtually all of the states, there is no small parameter which permits even the most primitive statistical analysis. The second and third branch are at least somewhat more accessible to analysis. But for the moment if one is to do any sort of "landscape phenomenology" one must adopt a hypothesis as to which branch of the landscape nature chooses (if any), and see if there is enough one can establish about the statistics of that branch to make definite predictions.

Despite these cautionary notes, as we have indicated, there are two arguments which might favor the intermediate branch (the problem of metastability on the supersymmetric branch and the suppression of the population of the third branch). In this paper, we will adopt the hypothesis that nature lies on this branch. We will argue that with some plausible assumptions - assumptions which one should be able to verify or disprove - this branch makes definite predictions for the spectra of gauginos and squarks and sleptons. The assumptions involve the detailed mechanisms by which the moduli are fixed and by which supersymmetry is broken, as well as the question of whether matter fields live on three branes or seven branes (in the IIB framework). The analysis of KKLT suggests two likely possibilities for the fate of the moduli. In both, all moduli have masses well above the scale of supersymmetry breaking. In the scenario actually put forward by KKLT, there are some moduli which are very light compared to the fundamental scale, but heavy compared to the supersymmetry breaking scale. We work out the scales in some detail, finding that the moduli can easily be several orders of magnitude more massive than the gravitino. In the second, all moduli have masses of order the fundamental scale. We consider two possible mechanisms for supersymmetry breaking which have been suggested for the landscape, and argue that they are likely to lead to similar spectra for the partners of ordinary fields. One is the anti-brane picture of [3], developed further particularly in [20, 21]; the second is dynamical supersymmetry breaking in a hidden sector 15, 8]. We argue that the qualitative features of the second are identical to those of the first, and that it is much easier to understand the nature of supersymmetry breaking in the latter case. We will see that gauginos typically are significantly lighter than squarks and sleptons. The precise hierarchy depends on whether there are light moduli and whether matter resides on three-branes or seven-branes.

As we will explain, with further work on the statistics of gauge groups in the landscape, 
one can hope to verify or refute these hypotheses. We will discuss briefly some ongoing efforts to address these issues.

Given that this branch predicts that gauginos are lighter than squarks and sleptons, it is natural to ask why, in nature, the scalars don't have mass approximately equal to the weak scale, while the gauginos have much smaller mass. Given the present limits on gaugino and higgs masses, the situation appears quite finely tuned. In the framework of the landscape, it is necessary to argue that there is some selection for large gaugino masses. The most plausible selection criterion is a presumed requirement of cold dark matter. If there is an $R$ parity (likely necessary to suppress proton decay) then the lightest of the gauginos is a natural dark matter candidate. It might be produced thermally or through decays of moduli or gravitinos [23, 22, 24]. Requiring that the dark matter be in the correct range makes interesting predictions for the gaugino and scalar masses.

In the following sections, we develop the phenomenology and cosmology of the intermediate scale branch. The next section discusses supersymmetry breaking in the KKLT picture, noting that susy breaking in hidden sectors is qualitatively similar to breaking by $\overline{D 3}$ branes. Section 3 outlines the possible phenomenologies, and the resulting hierarchies of masses. Section 4 explains how selection for the dark matter density could account for the apparent tuning of the Higgs mass in supersymmetric theories. Section 5 considers both predictions of this picture, and ways it might fail.

\section{The KKLT Picture}

In their original paper, KKLT proposed a picture in which the moduli are fixed and supersymmetry is broken, possibly with positive cosmological constant. We will, following KKLT, consider orientifolds of IIB theories on Calabi-Yau spaces. It is helpful to enumerate the basic features of the KKLT analysis; later we will discuss which of these features are expected to be general.

1. There are two sets of moduli, complex structure moduli, $z_{i}$, and Kahler moduli, $\rho$. Choosing a set of fluxes fixes the complex structure moduli, which obtain large masses. The effective action for the Kahler moduli includes a superpotential, which for large $\rho$ has the form

$$
W=W_{o}+a e^{i c \rho} .
$$

$W_{o}$ has a distribution which is flat for small $W_{o}$. This fact has been verified in explicit studies 6, 9], but it is not surprising: provided there is a dense set of states at small $W_{o}$, and provided that there is nothing special about $W_{o}=0$, the distribution function is non-singular and has a Taylor series expansion [9]. For small $W_{o}$, the potential has an AdS stationary point at large $\rho$, with unbroken supersymmetry.

2. Supersymmetry can be broken if $\overline{D 3}$ branes are present. This breaking will be small if the brane is located near a warped throat. It gives rise to an additional term in the potential for $\rho$,

$$
V(\rho)=m_{w a r p}^{4} \frac{1}{\rho^{3}}
$$


$m_{\text {warp }}^{4}$ is the $\overline{D 3}$ tension; it is small due to the warping in the throat. The distribution of $m_{\text {warp }}^{2}$ is also known (more precisely the distribution of warp scales is known 6 :

$$
\int d m_{\text {warp }} P_{\text {warp }}\left(m_{\text {warp }}\right)=\int \frac{d m_{\text {warp }}^{2}}{m_{\text {warp }}^{2} \ln \left(m_{\text {warp }}\right)}
$$

For some fraction of states, this leads to a cosmological constant which is small and positive. There have been a number of comments in the literature on the nature of supersymmetry breaking in the anti-brane picture, and there has been a good deal of confusion about whether the breaking should be viewed as spontaneous or explicit. For example, it has been suggested that this should be thought of as Fayet-Iliopoulos mechanism, but this is problematic. It is likely that the complications arise not only because the models are not particularly explicit, but because, in the warped geometry, one cannot simply write an effective lagrangian, near $m_{w a r p}$, including only a finite number of states.

3. These last objections can be addressed by considering, instead, states in the landscape in which supersymmetry breaking is due to the low energy dynamics of an additional gauge sector 15, 8, 36]. Given the assumption that chiral theories are common in the landscape, theories with such sectors are likely to exist and are probably numerous. As a model, one can consider the $(3,2)$ theory as a hidden sector. There is no problem in such a situation understanding the effective lagrangian. The longitudinal mode of the gravitino arises from the hidden sector. If one writes an effective lagrangian, the SUSY breaking in the visible sector appears explicit. From a phenomenological point of view, such models are essentially identical to the anti-brane theories. The distribution of supersymmetry-breaking scales is the same. The distribution of gauge couplings is roughly flat in $g^{2}$ [6]. This corresponds to a distribution of supersymmetry breaking scales,

$$
\int d m_{3 / 2} P\left(m_{m_{3 / 2}}\right)=\int \frac{d m_{3 / 2}^{2}}{m_{3 / 2}^{2} \ln \left(m_{3 / 2}\right)}
$$

just as from branes at warped throats. If this hidden sector lies on a $D 3$ brane, the potential behaves as $1 / \rho^{3}$, just as in the anti-brane picture. All of this is consistent with the notion that in some sense these two pictures are dual to each other. Many other features of the theory are easily understood in this picture. For example, stationary points with small cosmological constant are automatically local minima of the potential for $\rho$. This is because, if $\rho$ is large,

$$
\frac{\partial^{2} V}{\partial \rho^{2}} \approx e^{K}\left|\frac{\partial^{2} W}{\partial \rho^{2}}\right|^{2} g^{\rho \bar{\rho}}
$$

The hierarchies of masses depend on whether hidden and visible sector fields lie on $D 3$ or $D 7$ branes, and are readily determined in each case. We will enumerate the various possibilities in the next section. 
The KKLT analysis applies to theories with large $\rho$. In this case, there is, in some sense, a small parameter ${ }^{1}$. But we should note that the states with small $\rho$ are also potentially important, even if they may be more difficult to study ${ }^{2}$. One expects that there are many such states, associated either with large $W_{o}$ or with higher order terms in the superpotential. For these states, all of the moduli are typically quite heavy. Consider, first, supersymmetric states. If the cosmological constant is large, there is no low energy theory to discuss. If it is small, one can integrate out the massive fields to obtain an approximately Lorentz-invariant, supersymmetric lagrangian for the remaining light fields (at least the graviton and gravitino). The low energy theory will be characterized by a superpotential, $W_{o}$, and other parameters. By our earlier arguments, the distribution of $W_{o}$ (now obtained after integrating out all of the moduli) will still likely be nearly uniform. At small $\rho$, the geometric picture is not valid. But the statistics of supersymmetry breaking in the DSB picture are expected to remain the same; they rely only on some very weak assumptions about distributions of low energy couplings. So quite generally, we might expect the same distribution of $m_{3 / 2}$ to hold for both large and small $\rho$.

We will see that, in the large $\rho$ case, $1 / \rho$ serves as a small parameter. Numerically, it's value depends on dynamical details, but if the supersymmetry is broken within a decade or two of the TeV scale, we expect

$$
\rho \sim-a \ln \left(m_{3 / 2} / M_{p}\right) \approx a \times 35
$$

where $a$ is a number of order unity. It is worth keeping this in mind in our subsequent discussion.

\section{KKLT At Low Energies}

In this section, we focus on models with supersymmetry broken dynamically in a hidden sector. We will assume that all of the complex structure moduli are very massive. If the Kahler moduli are light, the low energy theory is described by $W_{o}$, a superpotential for $\rho$, and an action for the gauge and matter fields. If the Kahler moduli are massive, there is just $W_{o}$.

\subsection{Structure of Soft Breakings}

As a model, we will consider, as suggested above, a theory, such as the $(3,2)$ theory, coupled to supergravity. In [25], it was argued that in models with supersymmetry dynamically broken in a hidden sector, gauginos would be lighter than scalars. The argument was simple. Suppose that $W_{o}$ is adjusted to (nearly) cancel the cosmological constant, and that the scale of supersymmetry breaking is of order $\Lambda_{h i d}=m_{3 / 2} M_{p}$. In dynamical models, this is also the scale of the dynamics of the hidden sector. Theses models typically have no flat directions, so expectation values of fields are of this order; $F$ terms are of order $\Lambda_{\text {hid }}^{2}$. Finally, models of DSB typically have no gauge-singlet fields. Then, calling the

\footnotetext{
${ }^{1}$ The qualifier "some sense" is necessary because, in the IIB case, $\rho$ cannot be made arbitrarily large.

${ }^{2}$ We thank Shamit Kachru for stressing this point to us.
} 
visible matter sector fields $\phi_{i}$, and the $(3,2)$ sector fields $Z_{i}$, there are contributions to visible sector scalar masses from a variety of sources. These include the terms $\left|\frac{\partial K}{\partial \phi} W_{o}\right|^{2}$. Terms in the Kahler potential of the form $\phi^{\dagger} \phi Z^{\dagger} Z$ will also contribute to scalar masses. Gaugino masses, however, are more problematic. In the absence of singlets, one cannot write holomorphic gauge couplings which give rise to such masses. Anomaly mediation, then, would typically be the leading contribution 26, 27, 28].

This argument is correct for the case where there are no light moduli. However, if there are light moduli, these can acquire large $F$ terms, even in the absence of direct renormalizable couplings to the hidden sector. If such fields (denote them by $\mathcal{M}$ ), have Planck scale variation, then couplings such as

$$
K_{\mathcal{M} \phi}=\mathcal{M}^{\dagger} \mathcal{M} Z^{\dagger} Z+\mathcal{M} Z^{\dagger} Z+\ldots
$$

will generate order one shifts in $\mathcal{M}$, and order $m_{3 / 2} M_{p}$ values for the Kahler derivative $\frac{\partial K}{\partial \mathcal{M}} W$. So light moduli, in the presence of dynamical supersymmetry breaking, can generate $\mathcal{O}\left(m_{3 / 2}\right)$ masses for gauginos. Light moduli can have other effects as well.

In the IIB flux vacua, we have enumerated two possibilities. First, there may be no light moduli. In that case, the arguments of [25] would be correct, and gauginos would typically be light. Since the $W_{o}$ distribution is the same in both the light and heavy Kahler moduli cases, there is no reason to think that there are significantly more states of one type or the other. It is thus a phenomenological question whether one is on one branch or the other. One can advance some phenomenological arguments against the heavy moduli branch.

1. While gaugino masses may be lighter than squark and slepton masses, flavor changing neutral currents are not likely to be suppressed. (Note that there is no obvious anthropic argument which might select for, say, squark or slepton degeneracy).

2. Given that there is no apparent small parameter, it is not clear how coupling unification might emerge (though we do not have a compelling picture for how unification will emerge in the light modulus, large $\rho$, case, the existence of a small parameter which controls the coupling is promising.).

\subsection{Light Kahler Moduli; Hidden Sector on D3 Branes}

In the case where some of the Kahler moduli are light, a more detailed analysis is required. The form of the spectrum depends on whether the hidden sector and matter fields live on $D 3$ or $D 7$ branes. For simplicity, consider the case of a single complex structure modulus (the result generalizes to several moduli). If the hidden sector chiral fields, denoted by $z_{i}$, reside on $D 3$ branes, for large $\rho$ the Kahler potential is

$$
K=-3 \ln \left(i\left(\rho-\rho^{\dagger}\right)-z_{i}^{\dagger} z_{i}\right) .
$$

The gauge couplings in the hidden sector - and thus the scale of supersymmetry breaking, $\Lambda_{\text {hid }},-$ are independent of $\rho$. Suppose, as in the KKLT picture, one has found the solution of the condition

$$
D_{\rho} W=0
$$


For small $W_{o}$ in eqn. [2.1], $\rho$ is large, $\rho \sim-\ln \left(W_{o}\right)$. Now imagine "turning on" the hidden sector. This generates a term in the energy $\Lambda_{\text {hid }}^{4}$. For states with small cosmological constant, this is equal to $3\left|W_{o}\right|^{2} / \rho^{2}$. As a result, there is a supersymmetry-violating potential for the Kahler modulus,

$$
\begin{gathered}
V(\rho) \approx \frac{1}{\rho^{3}}\left(3\left|W_{o}\right|^{2}\right) \\
\approx m_{3 / 2}^{2} .
\end{gathered}
$$

We first estimate the mass of $\rho$, then the $\rho$ tadpole and $F$ term. The largest contribution comes from the supersymmetric part of the potential, eqn. 22.1. Because $\rho$ is large, we need only differentiate the exponential terms. Also, because (in the absence of the hidden sector) supersymmetry is unbroken, we have

$$
\frac{\partial W}{\partial \rho} \approx \frac{3}{\rho} W_{o}
$$

So the mass-squared of the $\rho$ field (recall the $\rho$ kinetic term is proportional to $1 / \rho^{2}$ is positive and hierarchically large compared to the gravitino mass:

$$
m_{\rho}^{2} \approx \rho^{2} m_{3 / 2}^{2} .
$$

In particular, it is large compared to $m_{3 / 2}$; the $\rho$ spectrum is approximately supersymmetric.

Treating $V_{h i d}$ as a perturbation, we can determine the shift in $\rho$ :

$$
\begin{gathered}
\Delta \rho=\frac{\partial V_{h i d}}{\partial \rho} / m_{\rho}^{2} \\
\approx \frac{1}{\rho} .
\end{gathered}
$$

Finally, the $\rho \mathrm{F}$ term follows from the equations of motion (keeping in mind the form of the $\rho$ kinetic term)

$$
F_{\rho} \approx W_{o} .
$$

Now we can ask the form of the soft breakings induced for visible sector fields. These depend, again, on whether the matter fields (chiral fields and gauge fields) reside on $D 3$ or $D 7$ branes. In the case of $D 3$ branes, gaugino masses receive no contributions from $F_{\rho}$, and the leading contributions to the gaugino masses are the anomaly mediated ones. The Kahler potential for the matter fields has a no-scale form. So the leading, $\mathcal{O}\left(m_{3 / 2}^{2}\right)$ terms vanish. The $F_{\rho}$ contribution to the masses is of order

$$
m_{\phi}^{2} \approx \frac{1}{\rho^{2}} m_{3 / 2}^{2} .
$$

If $\rho$ is of the order we have estimated earlier, then this is comparable to, and possibly somewhat larger than, the anomaly-mediated contribution to the squark and slepton masses. There can be additional contributions, arising from $\alpha^{\prime}$ corrections to the Kahler potential, as well as one loop effects in the low energy theory. The $\alpha^{\prime}$ corrections have been 
computed[29], and are of order $1 /(\rho)^{3 / 2}$. This correction has been estimated as between $10 \%$ and $1 \%$ [30, 31], corresponding to scalar masses of order $1 / 3-1 / 10$ of the gravitino mass.

If the Standard Model fields live on $D 7$ branes, the Kahler potential no longer has the sequestered form, and the squark and slepton masses are simply of order $m_{3 / 2}$. Gaugino masses are still suppressed, though now the coupling of $\rho$ to the gauge fields leads to a contribution of order $1 / \rho$ to gaugino masses beyond just the anomaly mediated contribution.

\subsection{Hidden Sector on D7 Branes}

If the hidden sector lies on $D 7$ branes, the scalings are different. Now $\Lambda_{\text {hid }}$ depends on $\rho$, $\Lambda_{h i d} \propto e^{a \rho}$. But the relation between $\Lambda_{h i d}$ and $W_{o}$ is also altered, because of the different structure of the Kahler potential:

$$
\Lambda_{h i d}^{4}=3\left|W_{o}\right|^{2} .
$$

So $\delta \rho \sim 1$. and the supersymmetry breaking mass term for $\rho$ is comparable in size to the non-supersymmetric term:

$$
\delta m_{\rho}^{2}=\rho^{2} m_{3 / 2}^{2}
$$

Correspondingly, $F_{\rho}$ is large:

$$
F_{\rho} \approx \rho m_{3 / 2} .
$$

Again, the masses of the visible sector fields depend on whether they reside on 3 branes of seven-branes. In both cases, the squark and slepton masses are of order $m_{3 / 2}^{2}$. If the gauge fields reside on 7 branes, because $\rho$ couples directly to the gauge fields and $F_{\rho}$ is large, the gaugino masses are of order $m_{3 / 2}$. If they reside on $D 3$ branes, again the principle contributions are the anomaly mediated ones.

\section{Phenomenology and Cosmology}

In the landscape, one would hope, on some principled grounds, to argue that some class of states are selected, and that this would lead to some definite phenomenological predictions. Such selection is likely to involve a combination of anthropic considerations, statistical features (e.g. far more of one type of state than another), and cosmological considerations. As the discussion of low energy supersymmetry in the introduction indicates, our current understanding is too primitive to make firm statements at present. But we can at least delineate some of the basic issues and speculate as to how predictions may emerge. In the case of low energy supersymmetry, we gave some arguments why supersymmetric states might be favored, and discussed expectations for the features of the statistics of supersymmetry breaking which follow from known features of the landscape. At a more detailed level, we advanced an argument that discrete $R$ symmetries (apart from $Z_{2}$ symmetries) are quite uncommon. Based on these considerations, we focussed most of our energy on the features of the intermediate scale branch.

Within this branch we have seen there are still a variety of choices: supersymmetry breaking could be due principally to fields on $D 3$ or $D 7$ branes, and the fields of the 
Standard Model could reside on one sort of brane or the other. We have also seen that there may be light moduli, or there may not. It might well be possible to determine that one or another of these phenomena is common or rare, just as we have argued that discrete $R$ symmetries are rare. For the moment, we can simply note that some of these possibilities are more readily reconciled with facts of nature than others.

There is an important question about which the landscape might shed some light. Low energy supersymmetry, in the post LEP II era, has become less attractive as an explanation of the hierarchy problem. Elaborate constructions are necessary to avoid tuning at the few per cent level. In the landscape framework, one would like to find a selection principle which accounts for this tuning. Perhaps the most plausible candidate is the density of dark matter (in combination with a selection for the scale of weak interactions). The dark matter density is closely tied, in modern theories of structure formation, to the features of galaxies. It has been suggested from time to time that anthropic considerations might fix the dark matter density. There is currently no convincing calculation which demonstrates this, but it is conceivable that this is the case, and, if so, we can ask where this might lead. In many of the models we have been studying in this paper, if the gaugino masses are fixed by dark matter considerations, to be in the several hundred $\mathrm{GeV}$ range, the squark and slepton masses will be 10's of TeV. The Higgs mass in a typical vacuum will also be of this order; only in a small fraction will the $W$ and $Z$ bosons be light. This, however, might be selected by other considerations, which have been widely discussed in the literature. In the rest of this section, we show that selection for the observed dark matter density and the weak scale leads to a cosmology for moduli and gravitinos which is compatible with known observations, as well as a predictive supersymmetry phenomenology, compatible with known facts.

\subsection{Cosmology}

We will focus in this section on the cosmology of the large $\rho$ (light $\rho$ ) case. Usually, the cosmology of moduli with masses comparable to the weak scale is problematic. These moduli come to dominate the universe long before recombination, and their decays tend to destroy the light elements produced during nucleosynthesis. One suggestion to resolve this dilemna 32] is to suppose that the moduli are relatively heavy. A $10 \mathrm{TeV}$ modulus might be expected to reheat the universe to about $10 \mathrm{Mev}$, restarting nucleosynthesis. Normally, this is said to involve an unnatural fine tuning. But we have just seen that in the KKLT model, $\rho$ is much heavier than the squarks and sleptons. We can easily imagine, in fact, that $m_{\rho}$ is of order $1000 \mathrm{TeV}$ or even larger! We have seen that this is not fine tuned, because the bulk of the $\rho$ mass is supersymmetric.

It is necessary that one produce baryons quite late in such a cosmology, but this could occur through A-D baryogenesis[33]. More serious are the questions of producing adequate dark matter and avoiding overproduction of gravitinos. In a similar framework, these issues have been dealt with in [23]. The reheat temperature at $\rho$ decay is approximately:

$$
T_{r h}=m_{\rho}\left(\frac{m_{\rho}}{M_{p}}\right)^{1 / 2}
$$




$$
\approx 0.2 \mathrm{GeV}\left(\frac{m_{3 / 2}}{10 \mathrm{TeV}}\right)^{3 / 2}(\rho / 35)^{3 / 2} .
$$

In the decays of $\rho$, direct production of both gauginos and gravitinos is suppressed by chirality. The fraction of the energy density initially in gravitinos is of order:

$$
\frac{\rho_{3 / 2}}{\rho_{\text {total }}}=C \frac{m_{3 / 2}^{3}}{m_{\rho}^{3}} \sim \rho^{-3} .
$$

Here we have adopted the notation of [23]; $C$ is plausibly of order 0.1-0.01. The gravitino lifetime, on the other hand, is $\rho^{3}$ longer than the modulus lifetime, corresponding to decay when the temperature has dropped by a factor $\rho^{3 / 2}$. The temperature at gravitino decay is thus of order:

$$
T_{3 / 2} \approx 1 \mathrm{MeV}\left(\frac{m_{3 / 2}}{10 \mathrm{TeV}}\right)^{3 / 2} .
$$

Even though the gravitinos still constitute a small fraction of the energy density (of order

$C \rho^{-3 / 2}$, one has to worry that gravitino decay products will destroy $D e, L i$, and other light elements [34]. However, as discussed at length in [23], there are a plausible range of parameters for which this is not a problem.

Our real question, however, is the dark matter density. As stressed in [23], gravitino decays will produce of order one LSP per decay. Suppose that the mass of the LSP is given by the anomaly mediated formula; then, one expects

$$
m_{L S P} \sim 3 \times 10^{-3} m_{3 / 2} .
$$

So the fractional density at $1 \mathrm{eV}$ is:

$$
\frac{\rho_{l s p}}{\rho_{\gamma}}=2 \times 10^{-1}\left(C / 10^{-2}\right)\left(\frac{m_{3 / 2}}{10 \mathrm{Tev}}\right)^{3 / 2}\left(\frac{\rho}{35}\right)^{-3 / 2} .
$$

So we see that we account for the dark matter density with an LSP which is in the right range to have escaped detection in accelerators, while the gravitino - and the scalar masses - are in the $\mathrm{TeV}$ range or higher.

\subsection{Phenomenology}

We will leave a thorough study of the phenomenology of these theories to further work. The basic point is that we have seen that selection for the dark matter density and the weak scale could account for the high masses of the squarks and sleptons, while implying that gauginos should be produced at the LHC. So the fact that LEP II did not discover supersymmetry is not surprising from this viewpoint.

\section{Conclusions: Selection effects, the little hierarchy, and other issues}

While low energy supersymmetry has many attractive features, experiments have narrowed significantly the possible parameter spaces of supersymmetric models, and it is generally believed that the lightness of the Higgs particle in supersymmetry constitutes a significant fine tuning problem. The cosmological argument we have presented above provides 
a possible resolution of this puzzle, provided we are willing to invoke a weak anthropic argument. The existence of structure probably requires the existence of cold dark matter, with a density (all other constants of nature held fixed) in a limited range. If the density is too low, fluctuations do not grow large until the universe is dark energy dominated. If the density is too high, it is possible (though by no means certain) that features of the resulting structure are inhospitable 37. Limits of the latter sort are subjects of debate; here we have simply assumed that such considerations limit the dark matter density to a narrow range. This in turn limits the lightest gaugino (the LSP in our scenario) to a narrow range of mass; squarks and sleptons are generically one or two orders of magnitude more massive. If we assume that the weak scale must also lie within a narrow range, then there would be a selection for the apparently tuned set of parameters which this model requires.

As usual, in any such discussion of anthropic selection, we cannot establish with any certainty that variation of several parameters - the gaugino masses, the inflationary fluctuation spectrum, the weak scale, and so on, one can't find other points consistent with the existence of observers, or whatever one feels is the correct selection criterion. We are here adopting the point of view that there may be selection for any parameter a drastic change of which would be devastating to the existence of life as we know it. We are establishing, at best, that it is plausible that a particular set of states in the landscape is preferred; we can then ask - as we have here - what are the consequences for experiments of such a preference.

With the assumptions we have made here, we see that a number of statements are robust. It is likely that in an order one fraction of states, squarks and sleptons are generally significantly more massive than gauginos. The moduli are quite heavy - either heavy enough that they restart nucleosynthesis, or so heavy that they play no role at all in low energy physics. In the former case, the squarks and sleptons are expected to be an order of magnitude or so more massive than the gauginos; in the latter, the gaugino masses arise from anomaly mediation, and the masses are separated by a full loop factor.

The resolution of the cosmological moduli problem, and the possible explanation of supersymmetric fine tuning are two attractive features of the picture we have developed. There are still many questions.

1. While squarks and sleptons can be significantly heavier than usually assumed, they are not heavy enough to resolve the flavor problems of supersymmetric theories. Some approximate degeneracy or alignment is still required. In the landscape, one must argue that this is either typical or that there is some effect which selects for such symmetries. One possibility is that there is some large class of Kahler potentials for which, at lowest order in $\rho$, squarks and sleptons are degenerate. The combination of somewhat heavier scalars and approximate degeneracy could resolve some of the questions of flavor.

2. Strong CP is a puzzle. There is no obvious, generic light axion candidate; because the $\rho$ field is approximately supersymmetric, there is no light pseudoscalar here. We 
are assuming that the lsp is the dark matter particle, so selection for dark matter is not likely to produce an axion.

3. Inflation and the Brustein-Steinhardt problem: it would be appealing if the field $\rho$ could somehow play the role of the inflaton. This may be possible, but it does not follow in an obvious way from the features of the $\rho$ dynamics which we have outlined. If a single Kahler modulus were to play the role of inflaton, then with the scales we have assumed here, the quantum fluctuations would be too small to account for structure. Multiple Kahler moduli might lead to hybrid inflation, but further tuning of potential parameters, at at least the $1 \%$ level, would be required to obtain adequate inflation and fluctuations. The $\rho$ fields also potentially suffer from the Brustein-Steinhardt problem. This might be solved by features of some early, high energy, period of inflation, along lines suggested in [39]. If some other field is responsible for inflation and dominates the energy for a time, the potential for $\rho$ can be appreciably altered in a way which dramatically slows the motion of the field.

There are many ways, as we have indicated, in which the ideas described here might fail. Perhaps the most dramatic is that the landscape may not exist [11], or alternatively that there might exist infinite numbers of states 10] whose existence might require significant rethinking of our basic understanding of string theory and what it might have to do with nature. But we believe we have outlined plausible predictions of a broad swath of states within the landscape. Further work could establish, or disprove, these ideas. It is important to do a more thorough analysis of the cosmology of these models as a function of the parameters $\rho$ and $m_{3 / 2}$, and this is in progress. One area for further study is the problem of dynamical supersymmetry breaking within the landscape. This is related to the problem of understanding distributions of gauge groups and matter content. Underpinning the structure we have studied in this paper is an assumption that hidden sectors without gauge singlets are generic, but this seems a question that one should be able to answer.

Acknowledgements: We thank A. Aguirre, N. Arkani-Hamed, T. Banks, A. Birkedal, S. Kachru, E. Silverstein and S. Thomas for conversations. This work supported in part by the U.S. Department of Energy.

\section{References}

[1] Precursors of the landscape idea appear in R. Bousso and J. Polchinski, JHEP 0006, 006 (2000) [arXiv:hep-th/0004134]; J. L. Feng, J. March-Russell, S. Sethi and F. Wilczek, Nucl. Phys. B 602, 307 (2001) [arXiv:hep-th/0005276]; S. B. Giddings, S. Kachru and J. Polchinski, Phys. Rev. D 66, 106006 (2002); [arXiv:hep-th/0105097], B. S. Acharya, arXiv:hep-th/0212294 and arXiv:hep-th/0303234.

[2] Much of the current interest in the landscape developed from the paper: S. Kachru, R. Kallosh, A. Linde and S. P. Trivedi, Phys. Rev. D 68, 046005 (2003) [arXiv:hep-th/0301240];

[3] L. Susskind, arXiv:hep-th/0302219. 
[4] Perhaps the most compelling rationale for taking seriously the idea of a landscape comes from considerations of the cosmological constant. See, in particular: S. Weinberg, Rev. Mod. Phys. 61, 1 (1989); J. Garriga and A. Vilenkin, Phys. Rev. D 61, 083502 (2000) [arXiv:astro-ph/9908115]; S. Weinberg, arXiv:astro-ph/0005265.

[5] The first efforts to understand the statistics of the landscape were developed in M. R. Douglas, arXiv:hep-ph/0401004;

[6] F. Denef and M. R. Douglas, JHEP 0405, 072 (2004) [arXiv:hep-th/0404116].

[7] F. Denef and M. R. Douglas, arXiv:hep-th/0411183.

[8] M. Dine, E. Gorbatov and S. Thomas, arXiv:hep-th/0407043.

[9] M. Dine, D. O’Neil and Z. Sun, arXiv:hep-th/0501214.

[10] O. DeWolfe, A. Giryavets, S. Kachru and W. Taylor, arXiv:hep-th/0505160.

[11] T. Banks, arXiv:hep-th/0412129.

[12] A. Saltman and E. Silverstein, JHEP 0411, 066 (2004) [arXiv:hep-th/0402135].

[13] K. Bobkov, arXiv:hep-th/0412239.

[14] B. S. Acharya, F. Denef and R. Valandro, arXiv:hep-th/0502060.

[15] T. Banks, M. Dine and E. Gorbatov, JHEP 0408, 058 (2004) [arXiv:hep-th/0309170]; and in [1].

[16] S. R. Coleman and F. De Luccia, Phys. Rev. D 21, 3305 (1980).

[17] O. DeWolfe, A. Giryavets, S. Kachru and W. Taylor, JHEP 0502, 037 (2005) [arXiv:hep-th/0411061].

[18] O. DeWolfe, in preparation.

[19] M. Dine and Z. Sun, arXiv:hep-th/0506246.

[20] K. Choi, A. Falkowski, H. P. Nilles, M. Olechowski and S. Pokorski, JHEP 0411, 076 (2004) [arXiv:hep-th/0411066].

[21] K. Choi, A. Falkowski, H. P. Nilles and M. Olechowski, Nucl. Phys. B 718, 113 (2005) [arXiv:hep-th/0503216].

[22] T. Moroi and L. Randall, Nucl. Phys. B 570, 455 (2000) [arXiv:hep-ph/9906527].

[23] K. Kohri, M. Yamaguchi and J. Yokoyama, Phys. Rev. D 72, 083510 (2005) [arXiv:hep-ph/0502211].

[24] R. Kitano and Y. Nomura, arXiv:hep-ph/0509221.

[25] I. Affleck, M. Dine and N. Seiberg, Nucl. Phys. B 256, 557 (1985).

[26] M. Dine and D. MacIntire, Phys. Rev. D 46, 2594 (1992) [arXiv:hep-ph/9205227].

[27] L. Randall and R. Sundrum, Nucl. Phys. B 557, 79 (1999) [arXiv:hep-th/9810155].

[28] G. F. Giudice, M. A. Luty, H. Murayama and R. Rattazzi, JHEP 9812, 027 (1998) [arXiv:hep-ph/9810442].

[29] K. Becker, M. Becker, M. Haack and J. Louis, JHEP 0206, 060 (2002)

[arXiv:hep-th/0204254]. 
[30] V. Balasubramanian, P. Berglund, J. P. Conlon and F. Quevedo, JHEP 0503, 007 (2005) [arXiv:hep-th/0502058].

[31] F. Denef, M. R. Douglas, B. Florea, A. Grassi and S. Kachru, arXiv:hep-th/0503124.

[32] T. Banks, D. B. Kaplan and A. E. Nelson, Phys. Rev. D 49, 779 (1994) [arXiv:hep-ph/9308292].

[33] I. Affleck and M. Dine, Nucl. Phys. B 249, 361 (1985); M. Dine, L. Randall and S. Thomas, Nucl. Phys. B 458, 291 (1996) [arXiv:hep-ph/9507453].

[34] M. Kawasaki, K. Kohri and T. Moroi, arXiv:hep-ph/0410287;

[35] M. Kawasaki, K. Kohri and T. Moroi, Phys. Rev. D 71, 083502 (2005) [arXiv:astro-ph/0408426].

[36] S. Kachru et al, to appear.

[37] A. Aguirre, private communication, and A. Aguirre and M. Tegmark, JCAP 0501, 003 (2005) [arXiv:hep-th/0409072]; M. Tegmark and M. J. Rees, Astrophys. J. 499, 526 (1998) [arXiv:astro-ph/9709058].

[38] G. Huey, P. J. Steinhardt, B. A. Ovrut and D. Waldram, Phys. Lett. B 476, 379 (2000) [arXiv:hep-th/0001112].

[39] M. Dine, Phys. Lett. B 482, 213 (2000) [arXiv:hep-th/0002047]. 